\title{
SEMIGROUPS OF HIGH RANK II DOUBLY NOBLE SEMIGROUPS
}

\author{
by EMILIA GIRALDES*
}

(Received 3rd July 1984)

\section{Introduction}

This paper is a sequel to [2]. By a semigroup of high rank we mean a semigroup such that for $s_{1} \neq s_{2},\left\langle S \backslash\left\{s_{1}, s_{2}\right\}\right\rangle \subset S$ (properly). Semigroups of high rank such that $\langle S \backslash\{s\}\rangle \subset S$ (royal semigroups) were classified in [2], where it was also shown that for a noble semigroup (i.e. a semigroup of high rank such that there exists a superfluous element $z$ in $S$ for which $\langle S \backslash\{z\}\rangle=S$ ) there exists either exactly one superfluous element or exactly two superfluous elements [2, Theorem 3.7].

The main results of [2] give structure theorems for singly noble semigroups (for which there is a unique superfluous element). The purpose of this paper is to describe the structure of doubly noble semigroups, i.e. semigroups $S$ in which there exist two distinct elements $z_{1}, z_{2}$ for which

$$
\left\langle S \backslash\left\{z_{1}\right\}\right\rangle=\left\langle S \backslash\left\{z_{2}\right\}\right\rangle=S
$$

In one important respect the description is easier than in the singly noble case, for a doubly noble semigroup must be a band [2, Theorem 3.7]. Moreover, if we express such a band in the standard way as a semilattice of rectangular bands, then not only must all rectangular bands be in $\mathbf{R Z} \cup \mathbf{L Z}$ [2, Lemma 2.1], but also the underlying semilattice must be a chain Theorem 3.9. A full structure theorem for doubly noble semigroups is therefore not very hard to obtain, and this is given as Theorem 3.10.

\section{Doubly noble semigroups}

Let $B$ be a doubly noble semigroup. Then $B$ is a band [2, Theorem 3.7] containing two elements $z_{1}, z_{2}$ such that $\left\langle B \backslash\left\{z_{1}\right\}\right\rangle=\left\langle B \backslash\left\{z_{2}\right\}\right\rangle=B$, but there do not exist distinct $s_{1}$ and $s_{2}$ such that $B \backslash\left\{s_{1}, s_{2}\right\}$ generates $B$. From [2, Section 3] we have

$$
\begin{gathered}
(\forall s, t \in B) \quad s t \in\left\{s, t, z_{1}, z_{2}\right\} \\
\left(\forall s, t \in B \backslash\left\{z_{i}\right\}\right) \quad s t \in\left\{s, t, z_{i}\right\} \quad i=1,2
\end{gathered}
$$

* This work was finished during a visit to St Andrews by the author, who acknowledges financial support from the Portuguese Instituto Nacional de Investigação Cientifica (Centro de Algebra de Lisboa). 
We also have

$$
\left(\forall s, t \in B \backslash\left\{z_{1}, z_{2}\right\}\right) \quad s t \in\{s, t\}
$$

for if $s t$ were equal to $z_{1}$ or $z_{2}$ it would follow that $B \backslash\left\{z_{1}, z_{2}\right\}$ generates $B$.

Next we have

Theorem 2.4 Let $B$ be a doubly noble band, with $\left\langle B \backslash\left\{z_{1}\right\}\right\rangle=\left\langle B \backslash\left\{z_{2}\right\}\right\rangle=B$. Then either

$$
\left(\exists x, y \in B \backslash\left\{z_{1}, z_{2}\right\}\right) \quad z_{1}=z_{2} x \quad \text { and } \quad z_{2}=z_{1} y
$$

or

$$
\left(\exists x, y \in B \backslash\left\{z_{1}, z_{2}\right\}\right) \quad z_{1}=x z_{2} \quad \text { and } z_{2}=y z_{1} .
$$

Proof. From (2.3) and from the fact that $\left\langle B \backslash\left\{z_{1}\right\}\right\rangle=B$ we must have either $z_{1}=z_{2} x$ or $z_{1}=x z_{2}$ for some $x$ in $B \backslash\left\{z_{1}, z_{2}\right\}$. Equally there exist $y$ in $B \backslash\left\{z_{1}, z_{2}\right\}$ such that $z_{2}=z_{1} y$ or $z_{2}=y z_{1}$. If

$$
z_{1}=z_{2} x \quad \text { and } \quad z_{2}=y z_{1}
$$

then

$$
z_{1}=z_{2} x=y z_{1} x=y z_{2} x^{2}=y z_{2} x=y z_{1}=z_{2}
$$

a contradiction. Similarly

$$
z_{1}=x z_{2} \text { and } z_{2}=z_{1} y
$$

lead to a contradiction. The result follows.

Notice now that (2.5) implies that $z_{1} \mathscr{R} z_{2}$, hence, since $B$ is a band,

$$
z_{1} z_{2}=z_{2}, \quad z_{2} z_{1}=z_{1}
$$

Similarly (2.6) implies $z_{1} \mathscr{L}_{z_{2}}$ and so

$$
z_{1} z_{2}=z_{1}, \quad z_{2} z_{1}=z_{2}
$$

In the former case we say that $B$ is a dexter doubly noble band, in the later case we say that $B$ is a sinister doubly noble band. In what follows we shall confine ourselves to the dexter case; results for the sinister case will always follow by duality.

Notice now that the elements $x$ and $y$ appearing in (2.5) must be distinct, if we had $z_{1}=z_{2} x, z_{2}=z_{1} x$ it would follow that

$$
z_{1}=z_{2} x=z_{2} x^{2}=z_{1} x=z_{2} .
$$

We thus deduce that if $B$ is a doubly noble semigroup, then $|B| \geq 4$. 
Theorem 2.8. Let $B$ be a dexter doubly noble band. Then

(i) for all $s$ in $B \backslash\left\{z_{1}\right\}$

$$
\begin{gathered}
s z_{1}=s \Rightarrow z_{1} s=z_{2} s=s z_{2}=s, \\
s z_{1}=z_{1} \Rightarrow z_{1} s \in\left\{s, z_{1}, z_{2}\right\}
\end{gathered}
$$

(ii) for all $s$ in $B \backslash\left\{z_{2}\right\}$

$$
\begin{gathered}
s z_{2}=s \Rightarrow z_{1} s=z_{2} s=s z_{1}=s, \\
s z_{2}=z_{2} \Rightarrow z_{2} s \in\left\{s, z_{1}, z_{2}\right\} .
\end{gathered}
$$

Proof. It will be sufficient to prove (i). So suppose first that $s z_{1}=s$. Then by (2.1)

$$
s z_{2} \in\left\{s, z_{1}, z_{2}\right\}
$$

now

$$
s=s z_{1}=s z_{2} z_{1}
$$

If $s z_{2}=z_{1}$, then $z_{1}^{2}=s$, a contradiction. If $s z_{2}=z_{2}$, then $z_{2} z_{1}=s$, again a contradiction. Hence $s z_{2}=s$. By (2.1) we have

$$
z_{1} s, z_{2} s \in\left\{s, z_{1}, z_{2}\right\}
$$

If $z_{1} s=z_{1}$, then

$$
z_{1}=z_{1} s=z_{1} s z_{2}=z_{1} z_{2}=z_{2}
$$

a contradiction. If $z_{1} s=z_{2}$ then

$$
z_{2}=z_{1} s=z_{1} s z_{1}=z_{2} z_{1}=z_{1}
$$

again a contradiction. Thus $z_{1} s=s$, and similarly $z_{2} s=s$. The second part of the statement (i) follows directly from (2.1).

Corollary. (i) For all $s$ in $B \backslash\left\{z_{1}\right\}$,

$$
z_{1} s \neq s \Rightarrow s z_{1}=z_{1}, s z_{2}=z_{2}
$$

(ii) For all $s$ in $B \backslash\left\{z_{2}\right\}$,

$$
z_{2} s \neq s \Rightarrow s z_{1}=z_{1}, s z_{2}=z_{2}
$$


Example. Let $B_{4}$ be the subsemigroup of $\mathscr{T}(\{1,2,3,4\})$

$$
\begin{array}{rlrl}
x & =\left(\begin{array}{llll}
1 & 2 & 3 & 4 \\
2 & 2 & 3 & 3
\end{array}\right) & y & =\left(\begin{array}{llll}
1 & 2 & 3 & 4 \\
1 & 1 & 4 & 4
\end{array}\right) \\
z_{1}=\left(\begin{array}{llll}
1 & 2 & 3 & 4 \\
3 & 3 & 3 & 3
\end{array}\right) & z_{2}=\left(\begin{array}{llll}
1 & 2 & 3 & 4 \\
4 & 4 & 4 & 4
\end{array}\right)
\end{array}
$$

This has the Cayley table

\begin{tabular}{c|cccc} 
& $x$ & $y$ & $z_{1}$ & $z_{2}$ \\
\hline$x$ & $x$ & $y$ & $z_{1}$ & $z_{2}$ \\
$y$ & $x$ & $y$ & $z_{1}$ & $z_{2}$ \\
$z_{1}$ & $z_{1}$ & $z_{2}$ & $z_{1}$ & $z_{2}$ \\
$z_{2}$ & $z_{1}$ & $z_{2}$ & $z_{1}$ & $z_{2}$
\end{tabular}

and is a dexter doubly noble band generated by $B \backslash\left\{z_{1}\right\}$ and $B \backslash\left\{z_{2}\right\}$. It illustrates the fact that the rather weak statement in the second parts of (i) and (ii) in Theorem 2.8 cannot be strengthened. If $s=x$ then

$$
s z_{1}=z_{1} \text { and } z_{1} s=z_{1} \text {, }
$$

if $s=y$ then

$$
s z_{1}=z_{1} \text { and } z_{1} s=z_{2}
$$

if $s=z_{2}$ then

$$
s z_{1}=z_{1} \text { and } z_{1} s=s .
$$

We now show that $B_{4}$ is a rather significant example.

Theorem 2.9. Let $B$ be a dexter doubly noble band generated by $B \backslash\left\{z_{1}\right\}$ and $B \backslash\left\{z_{2}\right\}$ and let $x, y$ be elements of $B \backslash\left\{z_{1}, z_{2}\right\}$ satisfying (2.5). Then $\left\{x, y, z_{1}, z_{2}\right\}$ is a subband of $B$ isomorphic to $B_{4}$.

Proof. We know that $z_{1} z_{2}=z_{2}, z_{2} z_{1}=z_{1}$ from (2.7). From (2.5) it follows that

$$
z_{1} x=z_{2} x^{2}=z_{2} x=z_{1}
$$

and similarly that $z_{2} y=z_{2}$. From the corollary to Theorem 2.8 it follows that

$$
x z_{1}=y z_{1}=z_{1} \quad x z_{2}=y z_{2}=z_{2} .
$$


From (2.3) it follows that $x y \in\{x, y\}$. Now $x y=x$ implies that

$$
z_{1}=z_{2} x=z_{2} x y=z_{1} y=z_{2}
$$

a contradiction. Hence $x y=y$. Similarly $y x=x$. The result is now clear.

\section{A structure theorem for doubly noble bands}

Following the pattern established in [2] we begin by examining the structure of a dexter doubly noble band with exactly two $\mathscr{J}$-classes.

Let us examine the following construction. Let $A$ and $Z$ be disjoint right zero semigroups where $|A| \geqq 2,|Z| \geqq 2$. Let $P$ be a proper subset of $A$ such that $|P| \geqq 1$ and let $z_{1}$ and $z_{2}$ be fixed elements of $Z$. Define a multiplication on $A \cup Z$ by the rules

$$
\begin{array}{ll}
a z=z & (z \in Z, a \in A) \\
z a=z & \left(z \in Z \backslash\left\{z_{1}, z_{2}\right\}, a \in A\right) \\
z_{1} a=z_{2} a=z_{1} & (a \in P) \\
z_{1} a=z_{2} a=z_{2} & (a \in A \backslash P) .
\end{array}
$$

It is possible to check directly that this is an associative multiplication. It is easy also to see that $S$ is a doubly noble band, with superfluous elements $z_{1}$ and $z_{2}$ and with two $\mathscr{J}$-classes, namely $A$ and $Z$. By analogy with the notational devices used in [2] we can write

$$
B=D N B_{R}^{R}\left(A, Z ; P ; \mathrm{z}_{1}, z_{2}\right)
$$

Notice that the example $B_{4}$ described following Theorem 2.8 is

$$
B_{4}=D N B_{R}^{R}\left(\{x, y\},\left\{z_{1}, z_{2}\right\} ;\{x\} ; z_{1}, z_{2}\right) .
$$

A sinister doubly noble band dual to (3.2) is easily described and is denoted by

$$
D N B_{L}^{L}\left(A, Z ; P ; z_{1}, z_{2}\right)
$$

We now prove

Theorem 3.4. Every doubly noble semigroup with two $\mathscr{J}$-classes is isomorphic to a semigroup of type (3.2) or (3.3).

Proof. Let $B$ be a doubly noble band with elements $z_{1}, z_{2}$ such that

$$
\left\langle B \backslash\left\{z_{1}\right\}\right\rangle=\left\langle B \backslash\left\{z_{2}\right\}\right\rangle=B .
$$


Suppose first that $B$ is dexter. Then $z_{1} \mathscr{R} z_{2}$ and so certainly $z_{1}, z_{2}$ are in the same $\mathscr{J}$ class $Z$, then $|Z| \geqq 2$. We know by [2, Lemma 2.1] that $Z$ is either left or right zero, by (2.7) it follows that $Z$ is right zero.

There exist $x, y$ in $B \backslash\left\{z_{1}, z_{2}\right\}$ such that $z_{1}=z_{2} x, z_{2}=z_{1} y$. If $x \in Z$ then

$$
x=z_{2} x=z_{1}
$$

a contradiction. Hence $x \notin Z$, and similarly $y \notin Z$. Hence $x, y \in A(|A| \geqq 2)$, the other $\mathscr{J}$ class of $B$. By Theorem 2.9, $\left\{x, y, z_{1}, z_{2}\right\}$ is a subband of $B$ isomorphic to $B_{4}$. Hence in particular $x y=y, y x=x$, and so $A$, which must be either left zero or right zero, is in fact right zero. $B$ is thus a two element chain of right zero semigroups $A$ and $Z$, with

$$
A Z \subseteq Z, Z A \subseteq Z
$$

For $a \in A$ and $z \in Z$ we have $a z=z^{\prime} \in Z$. In fact

$$
z^{\prime}=a z=a z^{2}=z^{\prime} z=z
$$

so we have

$$
a z=z \quad(a \in A, z \in Z)
$$

If $z \in Z \backslash\left\{z_{1}, z_{2}\right\}$ then by (2.3) and (3.5)

$$
z a \in\{z, a\} \cap Z .
$$

Thus

$$
z a=z \quad\left(a \in A, z \in Z \backslash\left\{z_{1}, z_{2}\right\}\right)
$$

Finally notice that by (2.1) and (3.5)

$$
z_{1} a \in\left\{a, z_{1}, z_{2}\right\} \cap Z=\left\{z_{1}, z_{2}\right\}
$$

If $z_{1} a=z_{1}$ then by (2.5)

$$
z_{2} a=z_{1} y a=z_{1} a=z_{1}
$$

(since $A$ is a right zero semigroup). Similarly if $z_{1} a=z_{2}$ then $z_{2} a=z_{2}$. Thus $A$ divides into complementary sets given by

$$
\begin{aligned}
P & =\left\{a \in A: z_{1} a=z_{2} a=z_{1}\right\} \\
A \backslash P & =\left\{a \in A: z_{1} a=z_{2} a=z_{2}\right\} .
\end{aligned}
$$


Both sets are non-empty, since $x \in P$ and $y \in A \backslash P$. Thus

$$
\begin{array}{ll}
z_{1} a=z_{2} a=z_{1} & a \in P \\
z_{1} a=z_{2} a=z_{2} & a \in A \backslash P .
\end{array}
$$

Comparing (3.6), (3.7) and (3.8) with (3.1) we see that

$$
B \simeq D N B_{R}^{R} \quad\left(A, Z ; P ; z_{1}, z_{2}\right)
$$

In obtaining a more general theory for doubly noble bands the following result is crucial.

Theorem 3.9. Let $B=\mathscr{B}\left[Y:\left\{E_{a}: \alpha \in Y\right\}\right]$ be a doubly noble band, expressed as $a$ semilattice $Y$ of rectangular bands. Then $Y$ is a chain.

Proof. Suppose not, and let $\alpha$ be a branch point of $Y$. Thus there exist $\beta, \gamma>\alpha$ such that $\beta \gamma=\alpha$. Since $E_{\beta} E_{\gamma} \subset E_{\alpha}$ it follows that one element $z$ of $E_{\alpha}$ can be expressed as a product $x y$ with $x \in E_{\beta}, y \in E_{y}$ and so $x \neq z, y \neq z$. Thus $\langle B \backslash\{z\}\rangle=B$. Hence $z \in\left\{z_{1}, z_{2}\right\}$ and so $\left\{z_{1}, z_{2}\right\} \in E_{\alpha}$. On the other hand $x, y \in B \backslash\left\{z_{1}, z_{2}\right\}$ and so $x y \in\{x, y\}$. Then $x y \in E_{\alpha} \cap\{x, y\}$ $=\emptyset$ and we have a contradiction.

Now let $B$ an arbitrary doubly noble semigroup and $E_{0}$ the $\mathscr{J}$-class of $B$ containing $z_{1}$ and $z_{2}$. Then $E_{0} \in \mathbf{R Z}$ and $B$ is a chain

$$
B=\mathscr{B}\left[Y:\left\{E_{\alpha}: \alpha \in Y\right\}\right]
$$

where there is at least one element $\alpha$ in $Y$, such that $\alpha>0$. There exist $x, y$ in $B \backslash E_{0}$ such that

$$
z_{2} x=z_{1}, z_{1} y=z_{2}
$$

and we have seen for any $x, y$ satisfying these equations we have $x y=y, y x=x$, giving $x \mathscr{R} y$. It follows that

$$
\left\{u \in B: z_{2} u=z_{1}\right\} \cup\left\{v \in B: z_{1} v=z_{2}\right\}
$$

is contained in a single $\mathscr{J}$-class, say $E_{\alpha}, \alpha>0$, for if $z_{1} u=z_{1}$, then $u \mathscr{R} y$, and if $z_{1} v=z_{2}$ then $v \mathscr{R} x$. Then $E_{\alpha} \in \mathbf{R Z}$ and the structure of the subband $E_{\alpha} \cup E_{0}$ is that of

$$
D N B_{R}^{R}\left(E_{0} ; E_{\alpha} ; P ; z_{1}, z_{2}\right) \text {. }
$$

Consider now the subband $E_{\gamma} \cup E_{0}$ where $\gamma>0 \quad \gamma \neq \alpha$. If $u \in E_{y}$ then $z_{1} u \neq z_{2}$ and so by (2.1)

$$
z_{1} u \in\left\{z_{1}, u\right\} \cap E_{0}=\left\{z_{1}\right\}
$$


Thus $z_{1} u=z_{1}$, and similarly $z_{2} u=z_{2}$. If $z \in E_{0} \backslash\left\{z_{1}, z_{2}\right\}$ then our previous argument using (2.3) gives

$$
z u \in\{z, u\} \cap E_{0}
$$

and so $z u=z$ for all $u \in E_{\gamma}$ and $z \in E_{0}$. Also for all $u \in E_{y}$ and all $z \in E_{0}, u z \in E_{0}$ and so

$$
u z=u z^{2}=(u z) z=z
$$

by the right zero property of $E_{0}$. Thus $E_{\gamma} \cup E_{0}$ has the structure of royal semigroup, as described in [2].

Every subband $E_{v} \cup E_{\mu}$, such that $v, \mu \neq 0$ is a royal band.

We now show that $\alpha$ covers 0 . For suppose that $\alpha$ does not cover 0 and let $b \in E_{\delta}$ where $0<\delta<\alpha$. Then both $E_{\alpha} \cup E_{0}$ and $E_{\alpha} \cup E_{\delta}$ are royal. Choose $x$ in $E_{\alpha}$ such that $z_{1}=z_{2} x:$ then

$$
z_{1}=z_{2} x=\left(z_{2} b\right) x=z_{2}(b x)=z_{2} b=z_{2}
$$

a contradiction.

We have therefore proved the converse half of the following theorem; where $\Omega$ denotes the class of all non-zero cardinal numbers.

Theorem 3.10. Let $(Y, \leqq)$ be a chain and let 0 be a fixed non maximal element of $Y$ and suppose that $Y$ contains an element 1 covering 0 . Let $M: Y \rightarrow \Omega$ and $H: Y \rightarrow\{R, L\}$ be maps and suppose that $M(0) \geqq 2, M(1) \geqq 2$ and $H(0)=H(1)=R$. Let $E_{\beta}, \beta \in Y$, be a set containing $M(\beta)$ elements and having right or left-zero semigroup structure according as $H(\beta)$ is $R$ or $L$. Let $B$ the disjoint union $B=\cup\left\{E_{\beta}: \beta \in Y\right\}$ and $z_{1}, z_{2} \in E_{0}$. Let $\emptyset \neq P \subseteq E_{1}$.

Extend the binary operation on $E_{\beta}, \beta \in Y$ to $B$ giving $E_{1} \cup E_{0}$ the structure

$$
D N B_{R}^{R}\left(E_{1}, E_{0} ; P ; z_{1}, z_{2}\right)
$$

and all other unions $E_{\beta} \cup E_{\gamma}$ the structure

$$
\operatorname{Roy}(\{\beta, \gamma\}, M|\{\beta, \gamma\}, H|\{\beta, \gamma\})
$$

(see [2, Theorem 2.2]). Then B is a dexter doubly noble band, denoted by

$$
D N B(Y ; M, H ; P) \text {. }
$$

Conversely, every dexter doubly noble band is isomorphic to one constructed in this way.

Proof. All that remains is to show that the multiplication described in $B$ is associative. Let $a \in E_{\alpha}, b \in E_{\beta}, c \in E_{\gamma}$, where $\alpha, \beta, \gamma$ are distinct elements of $Y$. By (3.12) the associativity is obvious from the properties of royal semigroups unless $\{0,1\} \subset\{\alpha, \beta, \gamma\}$.

If $\{\alpha, \beta, \gamma\}$ includes one element of $Y$ that is lower than 0 then the associativity is again clear. Since $E_{1} \cup E_{0} \backslash\left\{z_{1}, z_{2}\right\}$ is royal we need only consider the six cases. 
(i) $a \in\left\{z_{1}, z_{2}\right\}, \beta=1, \gamma>1$,

(ii) $a \in\left\{z_{1}, z_{2}\right\}, \beta>1, \gamma=1$,

(iii) $b \in\left\{z_{1}, z_{2}\right\}, \alpha=1, \gamma>1$,

(iv) $b \in\left\{z_{1}, z_{2}\right\}, \alpha>1, \gamma=1$,

(v) $c \in\left\{z_{1}, z_{2}\right\}, \alpha=1, \beta>1$,

(vi) $c \in\left\{z_{1}, z_{2}\right\}, \alpha>1, \beta=1$.

All the verifications are routine and a sample verification will suffice. In case (i) $z_{1} b \in\left\{z_{1}, z_{2}\right\} \subset E_{0}$ and so $b c=b$; hence

$$
\left(z_{1} b\right) c=z_{1} b=z_{1}(b c) .
$$

The other cases are similar.

If $|\{\alpha, \beta, \gamma\}|<3$ the associativity is obvious.

\section{REFERENCES}

1. A. H. Clifford, Semigroups admitting relative inverses, Ann. of Math. 42 (1941), 1037-1049.

2. E. Giraldes and John M. Howie, Semigroups of high rank, Proc. Edinburgh Math. Soc. 28 (1985), 13-34.

3. J. M. HowIE, An introduction to semigroup theory (Academic Press, London, 1976).

4. D. McLean, Idempotent semigroups, American Math. Monthly 61 (1954), 110-113.

5. M. Petrich, Lectures in semigroups (Akademie-Verlag, Berlin, 1977).

Departmento de Matemática

Faculdade de Ciencias

UNIVERSIDADE DE LISBOA

1300 LISBOA

Portugal 